\title{
Surgical and Radiological Assessment of Small Intestinal Neoplasms
}

\author{
Ahmed A. S. Salem1*, Mahoud H. El Shoieby1, Amr F. Mourad², \\ Hosam El-Din Galal Mohammad ${ }^{3}$ \\ ${ }^{1}$ Department of Surgical Oncology, South Egypt Cancer Institute, Assiut University, Assiut, Egypt \\ ${ }^{2}$ Department of Diagnostic Radiology, South Egypt Cancer Institute, Assiut University, Assiut, Egypt \\ ${ }^{3}$ Department of Diagnostic Radiology, Faculty of Medicine, Assiut University, Assiut, Egypt \\ Email: *ahmed_awad721@yahoo.com, elshoieby@live.co.uk
}

Received 14 January 2016; accepted 19 February 2016; published 22 February 2016

Copyright (C) 2016 by authors and Scientific Research Publishing Inc.

This work is licensed under the Creative Commons Attribution International License (CC BY).

http://creativecommons.org/licenses/by/4.0/

c) (i) Open Access

\begin{abstract}
Purpose: To highlight the role of multi-detector computed tomography in evaluation of small intestinal neoplastic lesions. Patient and Methods: Thirty patients suspected to have small bowel neaoplasms were examined with 64 MDCT and underwent surgical exploration. Result: Contrast enhanced MDCT enterography easily diagnosed twenty patients with small intestinal neoplasms which were confirmed with surgical exploration and histopathological results. Conclusions: Our study has been proved that MDCT can be used as a front-line imaging modality for detection of small bowel neoplasms, regarding its ability to show intraluminal, mural and extraintestinal lesions with their characteristic density and features to successfully differentiate between the different neoplasms and sometimes stage them. However, operative exploration with curative resection or biopsy is still the confirmatory diagnostic method.
\end{abstract}

\section{Keywords}

Contrast Enhanced (CE), Multidetector Computed Tomography (MDCT), Enterography, Surgical Exploration, Small Intestinal Neoplasms

\section{Introduction}

There is a vast array of pathologic processes that occurs in the small bowel and mesenteric vasculature [1]. Imaging of the small bowel is challenging technically. Because the organ is long and serpentine, a large field of view and a large volume are needed to display in entirety. Another problem for imaging is motion, both intrinsic

${ }^{*}$ Corresponding author.

How to cite this paper: Salem, A.A.S., El Shoieby, M.H., Mourad, A.F. and El-Din Galal Mohammad, H. (2016) Surgical and Radiological Assessment of Small Intestinal Neoplasms. Journal of Cancer Therapy, 7, 96-106.

http://dx.doi.org/10.4236/jct.2016.72011 
motion of peristalsis and the positional changes caused by breathing. These two motion patterns can be additive and lead to a complex movement of individual bowel loops, making their tracing very difficult. In addition, because small bowel diseases have a low incidence, their appearance is less well known and there is an increased risk of missing them. Ever most of the common diseases in the small bowel, early changes are subtle making their diagnoses difficult [2]. Enteroscopy shows promise for the study of the small bowel, but this technique is invasive and cannot explore the totality of the small bowel [3]. Recent innovations, including capsule endoscopy and Magnetic Resonance Imaging (MRI), have emerged as alternative small bowel imaging techniques that can be performed without ionizing radiation [1]. However, MRI appears less accurate and less sensitive than CT in detection of bowel wall thickening, abnormal bowel wall enhancement, and adenopathy. Also, capsule endoscopy has its drawbacks of capsule obstruction by bowel strictures, capsule retention and battery failure in prolonged transit. It may as well provide false-negative results if there is rapid peristalsis at a lesion site or if there is bowel angulations at a lesion that impairs the camera view, and equivocal results in the presence of excess luminal fluid [4] and [5]. Currently, the availability of Multidetector computed tomography (MDCT) that acquires isotropic data, oral contrast agents, and administration techniques that improve small-bowel distention coupled with multiplanar and 3D imaging processes, have allowed improved depiction and characterization of small bowel pathology. Thin collimation and fast scanning allow coverage of the entire abdomen within a single suspended respiration phase. It has advantages over classic helical CT in the imaging of the mesenteric vasculature and of the small bowel. It can identify and stage most of the common diseases of the small bowel. It has the needed sensitivity, specificity, and availability for a front-line diagnostic method for almost all small bowel diseases. It can also detect ischemic changes in the affected bowel loops and mesenteric vessels [1] [2] [4]. MDCT with curved planar reformation clearly demonstrates the full extents of the mesenteric venous thrombus and the infarcted bowel loops [6] MDCT technologies can reveal potential bleeding sources and simultaneously depict morphological changes in the abdomen, such as intestinal tumors [7]. MDCT can be used to evaluate abnormalities in the bowel wall, mesentery, adjacent structures, and vasculature [8] [9]. The thinner collimation possible with MDCT along with oral administration of water and intravenous bolus of contrast material may improve the sensitivity of CT for depicting small-bowel tumors as it has the potential to demonstrate intraluminal, mural, and extra intestinal abnormalities [9]. MDCT is changing the paradigm for diagnosing small bowel disease by becoming the first diagnostic line for almost all small bowel diseases [2].

\section{Patients and Methods}

This is a prospective study which included 30 patients (23 males and 7 females) suspected to have small bowel neoplasms had undergone multidetector CT examination in Radiodiagnosis Department of Assiut University during the period from September 2013 till September 2015 and most of the cases are explored at surgical oncology department of south Egypt Cancer institute. The study was approved by the ethical committee of our hospital (with a patient informed consent) Twenty out the total number underwent surgical exploration for the purpose of confirmation of diagnosis or to relieve the intestinal obstruction. Their age ranged from 15 years to 70 years with a mean of 45.5 years. MDCT enterography was done to all patients MDCT enterography was preceded by other small bowel investigations such as plain radiography, and abdominal ultrasonography. All patients had a low-residue diet, ample fluids, laxative on the day prior to the examination, and they fasted for 4 hours before the examination. Our patients administered water as a neutral oral contrast agent so that the degree and pattern of small bowel enhancement can be well analyzed. At the imaging centers, patients ingested a volume of $1450 \mathrm{ml}$ water over a 40-minute period. On arrival at the imaging center, patients ingested $500 \mathrm{ml}$, ten minutes later patients ingested another $500 \mathrm{ml}$. Twenty minutes later patients ingested $225 \mathrm{ml}$. Finally, on entering the scanning room, the patient drank a $225 \mathrm{ml}$ of water to distend the stomach and duodenum. Intravenous contrast enhancement is essential on performing MDCT enterography so that the bowel wall is well-visualized. A 20-gauge canula was inserted into an arm vein and a $1.5 \mathrm{ml} / \mathrm{Kg}$ of iodinated contrast material (Ultravist 360 $\mathrm{mg}$ or Scanlux $300 \mathrm{mg}$ ) was injected at a rate of $4 \mathrm{ml} / \mathrm{sec}$ using an automated power injector. The delay between the start of the IV contrast injection and the start of the helical scanning was approximately 25 seconds to achieve the arterial phase, and 60 seconds for the delayed phase (also named enterography phase where the small bowel wall enhancement was optimum). Images were obtained from the dome of the liver to the lower margin of the symphysis pubis during a single breath-hold. Our patients were imaged with a 64-MDCT scanner (Toshiba Acquillion-Japan) using the following scanning parameters: 
- $64 \times 0.75 \mathrm{~mm}$ detector configuration.

- 600 millisecond gantry rotation.

- $0.8 \mathrm{~mm}$ slice width.

- 16 volume-pitch.

- $120 \mathrm{kVp}$.

- 180 effective mAs.

- 6 seconds total scan time.

- A dose modulator, which automatically decreases the radiation exposure to thinner areas of the patient, was used and could reduce the dose up to $30 \%$. The technologist generated a set of axial 3-mm sections and a set of 3-mm thick coronal multiplanar reformatted images at 3-mm intervals encompassing the entire bowel using the suitable reconstruction parameters. The thin slices were sent to the workstation, where they were available for us to view in 3D volume-rendering or maximum-intensity-projection displays. Different small-bowel abnormalities diagnosis was based upon the fore mentioned seven criteria, namely:

1) Pattern of enhancement.

2) Length of involvement.

3) Degree of thickening.

4) Whether the thickening is symmetric or asymmetric.

5) Location of the lesion along the course of the small bowel.

6) Location of the lesion in the wall of the small bowel.

7) Associated abnormalities in the mesentery and vessels.

\section{Results}

This study included 30 patients (2 males (76.7\%) and 7 females (23\%)). Their age ranged from 15 days to 70 years with a mean of 45.5 years. All patients had proved to be small intestinal malignant neoplasms documented by final histopathogical examination through surgical exploration or guided biopsy results. 18 (60\%) patients with lymphoma and 10 of them underwent surgical exploration and resection anastomosis was done (Figure 1), surgery was also carried out in 8 (26.6\%) patients with malignant gastrointestinal stromal tumors (GIST), 3 (10\%) patient with duodenal adenocarcinoma and 1 (3.3\%) patient with metastatic tumor (Table 1).

Twenty-four patients of the neoplastic group complained of recurrent abdominal pain which was dull-aching in character. It was epigastric in three of the twelve lymphoma patients while it was diffuse in the rest. The abdominal pain was acute and associated with absolute constipation and vomiting that was indicative of acute intestinal obstruction in the last malignant GIST patient.

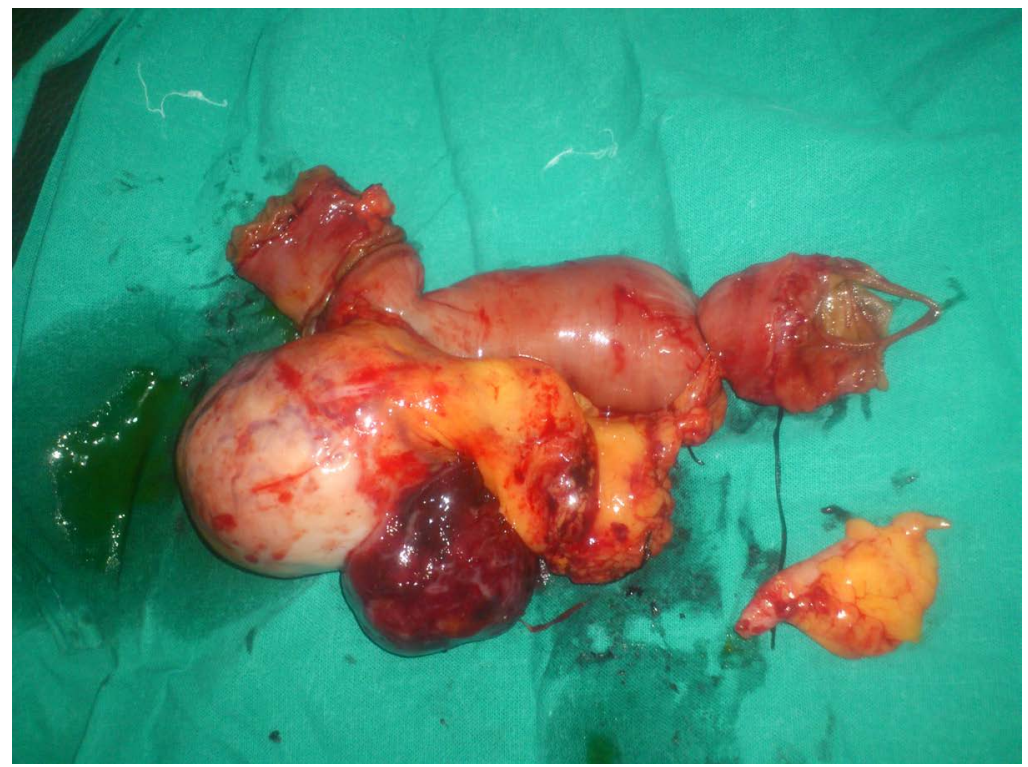

Figure 1. Postoperative specimen showing resected segment of small intestine. 
Table 1. Types of malignant neoplasms versus no. of patients.

\begin{tabular}{cc}
\hline MDCT diagnosis & No. of patients \\
\hline Lymphoma & 18 \\
Malignant GIST & 8 \\
Duodenal adenocarcinoma & 3 \\
Metastasis & 1 \\
\hline
\end{tabular}

Twenty-one patients complained of loss of weight. Nine of the patients complained of associated abdominal distension, four complained of associated melena, two complained of night fever and drenching sweats and another two complained of constipation.

\subsection{MDCT Findings in Lymphoma Patients}

This group of patients included 18 patients (14 males and 4 females).

The age of this group of patients ranged from 15 years to 70 years (Table 2). We used as the basis for diagnosis of small bowel diseases. The eighteen patients' small bowel showed segmental $(6-40 \mathrm{~cm})$ asymmetric thickening of the wall which involved the submucosa of the small intestinal wall forming a mass with the characteristic feature of aneurysmal wall dilatation without intestinal obstruction. The mucosa of the small intestine (other than the duodenum) is seen involved in thirteen (72.2\%) patients. Fifteen (83\%) of the patients' small bowel showed marked wall thickening, while the other three (17\%) showed moderate wall thickening. Only five (27.8\%) of the patients showed heterogeneous wall enhancement, while the rest (72.2\%) showed homogeneous wall enhancement. Lymphoma was located in the ileum in nine (50\%) patients, in the jejunum in five (27.8\%) patients, in the duodenum (16.7\%) in three patients and in the duodenum and jejunum in the last (5.6\%) patient. Associated enlarged mesenteric lymph nodes were found in thirteen (72.2\%) patients and engorged vessels in three (16.6\%) patients (Table 3, Figure 2 and Figure 3).

Table 3 summarizes the MDCT criteria of Lymphoma in the 18 patients. The table shows that the common criteria of Lymphoma in our study were marked asymmetric segmental wall thickening with homogenous wall enhancement most commonly involving the mucosa and submucosa of the ileum, most commonly associated with enlarged mesenteric lymph nodes. Pathological biopsy of the eighteen patients confirmed the MDCT diagnosis and revealed Non-Hodgkin Lymphoma. Two of the eighteen patients were diagnosed as recurrent Non-Hodgkin Lymphoma as they were previously treated surgically by resection anastomosis of the small intestinal lymphomatous lesion. Six (33.3\%) of the patients MDCT findings were associated with ascites, three (16.6\%) with malignant mass in the stomach and one (5.6\%) with multiple malignant-featuring hepatic focal lesions. Two of the patients were imaged by abdominal ultrasonography prior to MDCT examination which showed the small intestinal mass or thickening with associated enlarged lymph nodes in different groups mainly the mesenteric one. One of the Lymphoma patients was a male patient aged 18 years old complained of recurrent abdominal pain, abdominal distension, chronic watery diarrhea and loss of weight for about 5 years. This patient was diagnosed according to MDCT criteria and confirmed by biopsy as gastric and small bowel non-Hodgkin lymphoma. However, his small bowel also showed the criteria of Celiac disease. These criteria were moderate diffuse symmetric wall thickening with target appearance of enhancement. The submucosa of jejunum and ileum was involved. Associated multiple enlarged mesenteric lymph nodes were found. These findings were associated with marked ascites and the characteristic Celiac disease features that are multiple intussusception points and fold reversal.

\subsubsection{MDCT Findings in Gastrointestinal Stromal Tumors (GIST) Patients}

This group included six male and two female patients Based upon the seven MDCT criteria we used as the basis for diagnosis of small bowel neoplasms, the eight patients' small bowel showed asymmetric wall thickening forming an exophytic mass with heterogenous wall enhancement and involvement of the submucosa. Five of the patients' small bowel showed marked wall thickening and three showed moderate thickening. Ileum was involved in three patients while jejunum was involved in the other five. Only two patient's lymph nodes were enlarged (Table 4). 
Table 2. Age groups of Lymphoma patients Based upon the seven MDCT criteria.

\begin{tabular}{cc}
\hline Age group & No. of patients \\
\hline 1 - 20 years & 4 patients \\
$21-40$ years & 5 patient \\
$41-60$ years & 5 patients \\
$61-80$ years & 4 patient \\
\hline
\end{tabular}

Table 3. MDCT criteria of lymphoma.

\begin{tabular}{|c|c|c|c|c|c|c|c|}
\hline $\begin{array}{r}\begin{array}{r}\text { MDCT } \\
\text { criteria }\end{array} \\
\text { Patients }\end{array}$ & $\begin{array}{l}\text { Pattern of } \\
\text { enhancement }\end{array}$ & $\begin{array}{l}\text { Length of } \\
\text { involvement }\end{array}$ & $\begin{array}{l}\text { Degree of } \\
\text { thickening }\end{array}$ & $\begin{array}{c}\text { Symmetric or } \\
\text { asymmetric thickening }\end{array}$ & $\begin{array}{l}\text { Location along the } \\
\text { small bowel }\end{array}$ & $\begin{array}{l}\text { Location within } \\
\text { the wall }\end{array}$ & $\begin{array}{l}\text { Mesentery and } \\
\text { lymph nodes }\end{array}$ \\
\hline M70Y & homog & segmental & marked & asymm & Duod,jej & Submu & Mes. L.N. \\
\hline M18Y & homog & segmental & moderate & asymm & jejunum & Mucosa, Subm & Mes. L.N. \\
\hline M54Y & homog & segmental & marked & asymm & ileum & Mucosa, Subm & - \\
\hline F16Y & homog & segmental & marked & asymm & ileum & Mucosa, Subm & Mes. L.N. \\
\hline M21Y & homog & segmental & marked & asymm & duod. & Subm & Mes. L.N. \\
\hline F17Y & heterog & segmental & marked & asymm & ileum & Mucosa, Subm & Mes. L.N. \\
\hline M57Y & homog & segmental & marked & asymm & duod. & Subm & Mes. L.N. \\
\hline M15Y & heterog & segmental & marked & asymm & ileum & Mucosa, Subm & Mes. L.N. \\
\hline M50Y & homog & segmental & marked & asymm & ileum & Mucosa, Subm & - \\
\hline M30Y & homog & segmental & marked & asymm & jejunum & Mucosa, Subm & - \\
\hline M15Y & heterog & segmental & marked & asymm & ileum & Mucosa, Subm & Mes. L.N. \\
\hline M23Y & homog & segmental & moderate & asymm & jejunum & Mucosa, Subm & Mes. L.N. \\
\hline F35Y & Homog. & segmental & marked & asymm & jejunum & Mucosa, Subm & ----- \\
\hline M42Y & Heterog. & segmental & moderate & asymm & ileum & Mucosa, Subm & Mes. L.N \\
\hline M55Y & Homog. & segmental & Marked & asymm & ileum & Mucosa, Subm & Mes. L.N \\
\hline M65Y & heterog & segmental & Marked & asymm & jejunum & Mucosa, Subm & --- \\
\hline M66Y & Homog. & segmental & Marked & asymm & ileum & Mucosa, Subm & Mes. L.N \\
\hline M69Y & homog & Segmental & moderate & Asymm & duod & Mucosa-submuc & Mes. L.N \\
\hline
\end{tabular}

Table 4. MDCT criteria of GIST patients.

\begin{tabular}{|c|c|c|c|c|c|c|c|}
\hline Patients $\begin{array}{r}\text { MDCT } \\
\text { criteria }\end{array}$ & $\begin{array}{c}\text { Pattern of } \\
\text { enhancement }\end{array}$ & $\begin{array}{l}\text { Length of } \\
\text { involvement }\end{array}$ & $\begin{array}{l}\text { Degree of } \\
\text { thickening }\end{array}$ & $\begin{array}{c}\text { Symmetric or } \\
\text { asymmetric thickening }\end{array}$ & $\begin{array}{l}\text { Location along the } \\
\text { small bowel }\end{array}$ & $\begin{array}{l}\text { Location within } \\
\text { the wall }\end{array}$ & $\begin{array}{l}\text { Mesentery and } \\
\text { lymph nodes }\end{array}$ \\
\hline M19Y & heterog & segmental & marked & asymm & ileum & Submu & \\
\hline M70Y & heterog & focal & marked & asymm & jejunum & Submu & Mes. L.N. \\
\hline M68Y & heterog & segmental & moderate & asymm & jejunum & Submu & \\
\hline M65Y & heterog & segmental & marked & asymm & ileum & submu & ----------- \\
\hline M48Y & heterog & focal & moderate & asymm & Jejunum & Submu & -------- \\
\hline F70Y & Heterog & Segmental & Moderate & Asymm & Ileum & Submuc & ------ \\
\hline F45Y & Hetrog & Focal & Marked & Asymm & Jejunum & Submuc & Mes. L.N \\
\hline M57Y & Hetrog & Segmental & Moderate & asymm & jejunum & Submuc & \\
\hline
\end{tabular}




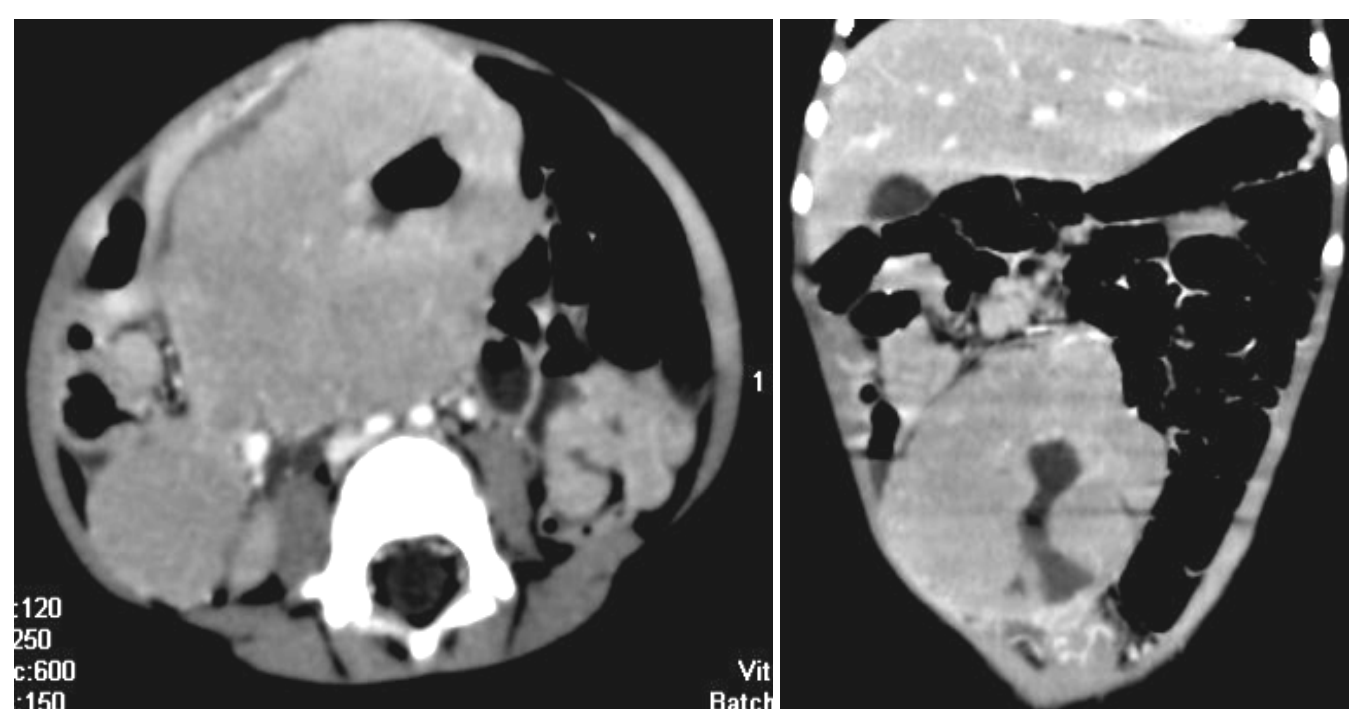

(a) Axial and coronal images

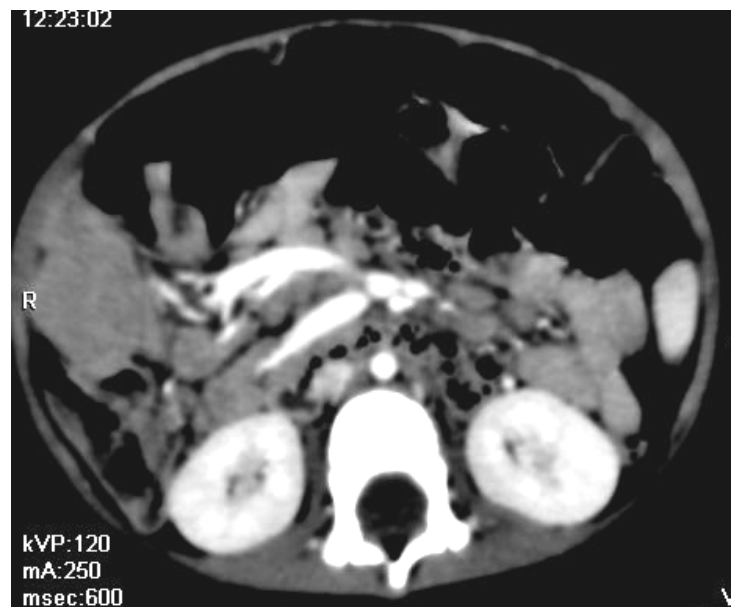

(b) Axial image

Figure 2. A 3 years old male patient with ileocecal junction lymphoma. MDCT showed segmental asymmetric marked wall thickening with heterogenous enhancement (white arrows) (a). The mucosa and submucosa were involved associated with multiple enlarged mesenteric lymph nodes showing the "sandwich sign" (white circle) (b).

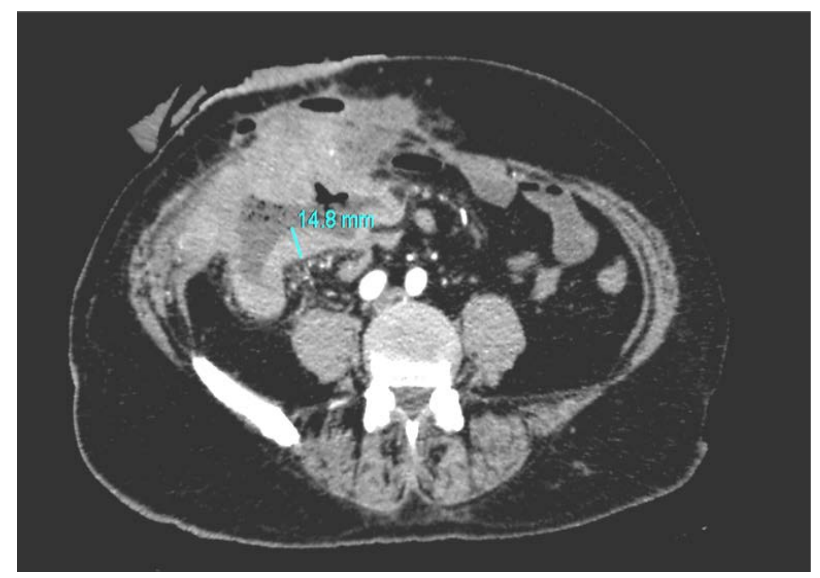

(a) Axial image 

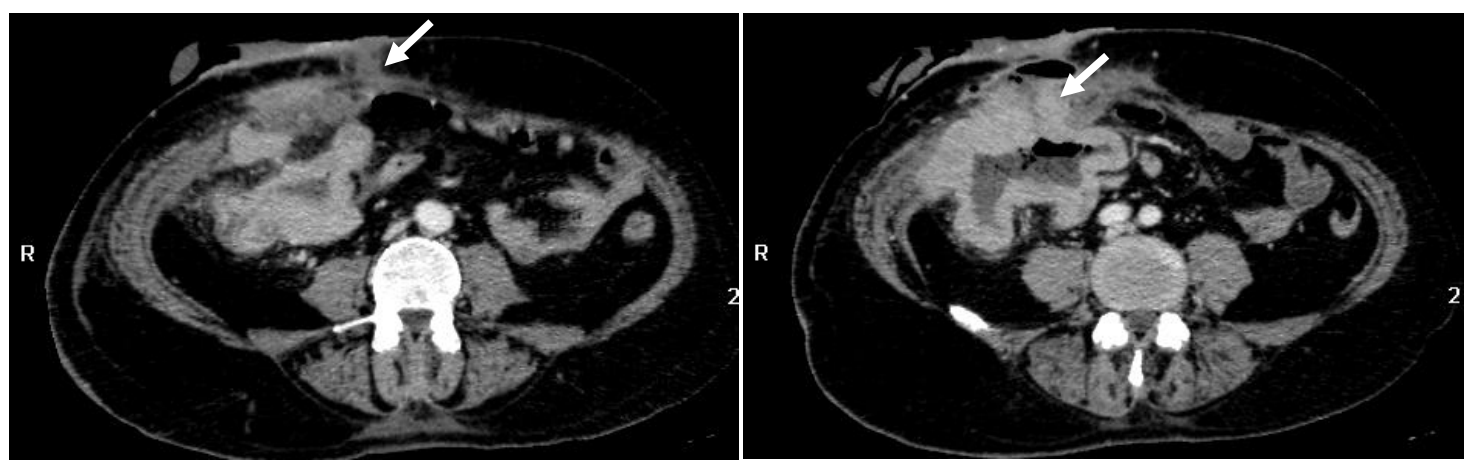

(b) Axial image

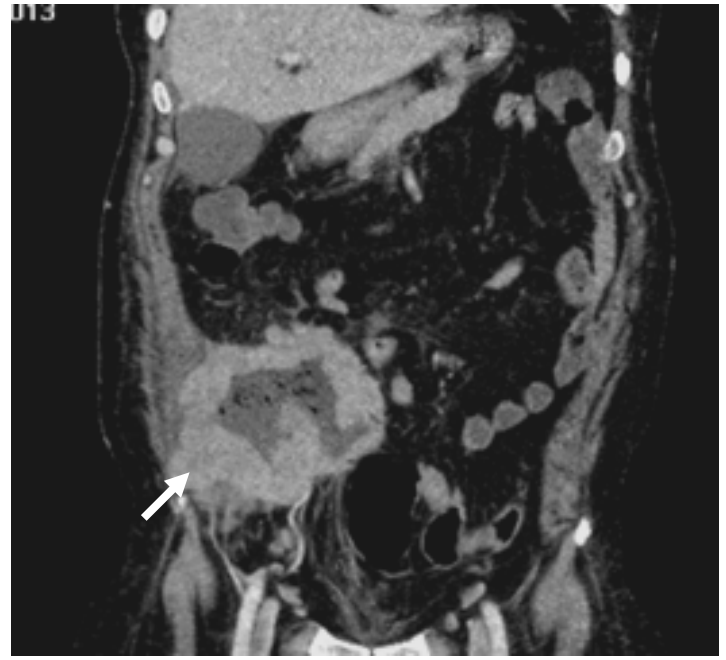

(c) Coronal image

Figure 3. A 50 years old male patient with recurrent ileal lymphoma with skin fistula. MDCT showed segmental asymmetric marked wall thickening with homogenous enhancement (a). The mucosa and submucosa of the ileum were involved showing the characteristic aneurysmal dilatation without intestinal obstruction. (white arrow) (c). The involved ileal wall in the right iliac fossa was seen infilterating the anterior abdominal wall muscles, subcutaneous tissue and skin resulting in a skin fistula. (white arrows) (b).

Four patient's MDCT findings were associated with small intestinal obstruction. The fifth was associated with multiple malignant hepatic focal lesions. The last three was associated with multiple peritoneal deposits. The pathological biopsy results in the eight patients revealed malignant spindle cell GIST. Two of the patients were imaged by abdominal ultrasonography prior to MDCT examination. The first one showed a mass lesion at the distal ileum with proximal small intestinal loops dilatation. The last one showed a small intestinal mass.

\subsubsection{MDCT Findings in Primary Duodenal Adenocarcinoma Patient}

There were two females and one male patients. Abdominal ultrasonography was done prior to MDCT examination that revealed multiple hepatic focal lesions. MDCT findings are summarized in (Table 5).

The small bowel of these patients showed focal asymmetric moderate wall thickening with heterogenous wall enhancement. This thickening involved the mucosa and submucosa of the associated with enlarged mesenteric lymph nodes, but associated with gastric wall thickening, multiple malignant hepatic focal lesions and multiple enlarged porta hepatis lymph nodes. This gastric wall thickening which was seen extending to the duodenum was proved to be adenocarcinoma by biopsy report.

This patient was a male patient aged 34 years old. The patient had done a Whipple operation for a pancreatic head adenocarcinoma infiltrating the duodenum 5 months ago. Post-contrast CT was done 1 month ago prior to this MDCT examination to reveal a jejunal mass with incomplete obstruction. MDCT findings are summarized in (Table 6 and Figure 4). 


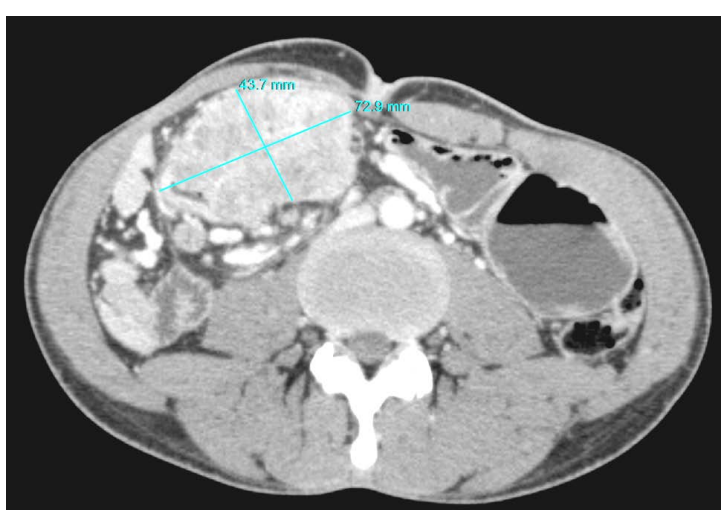

(a) Axial image (enterography phase)

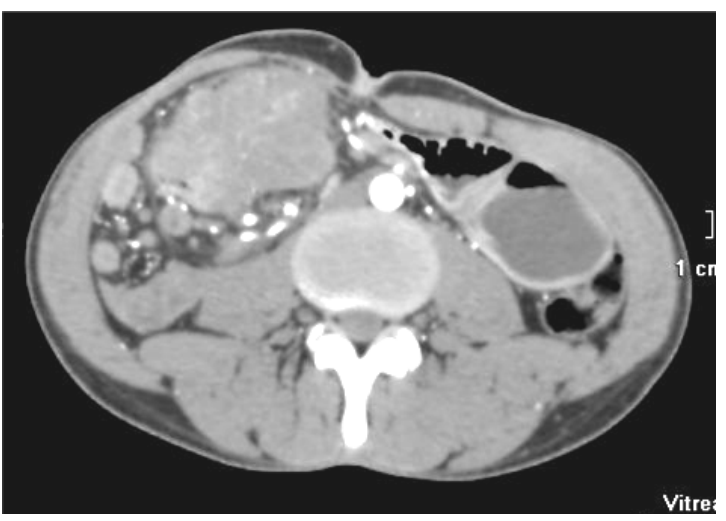

(b) Axial image (arterial phase)

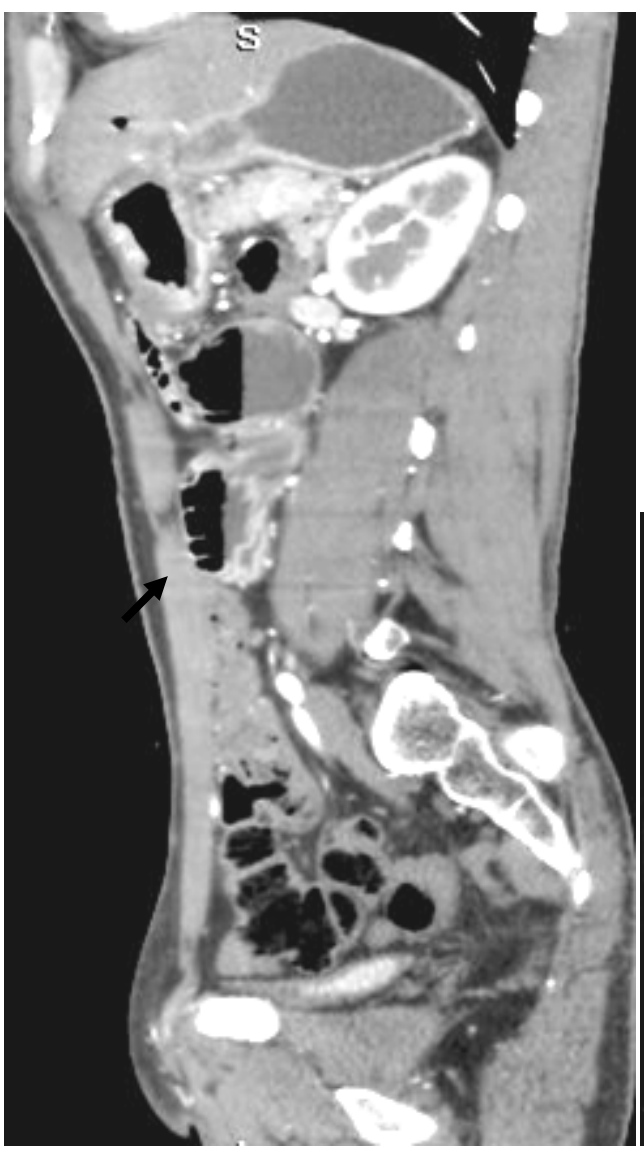

(c) Coronal image

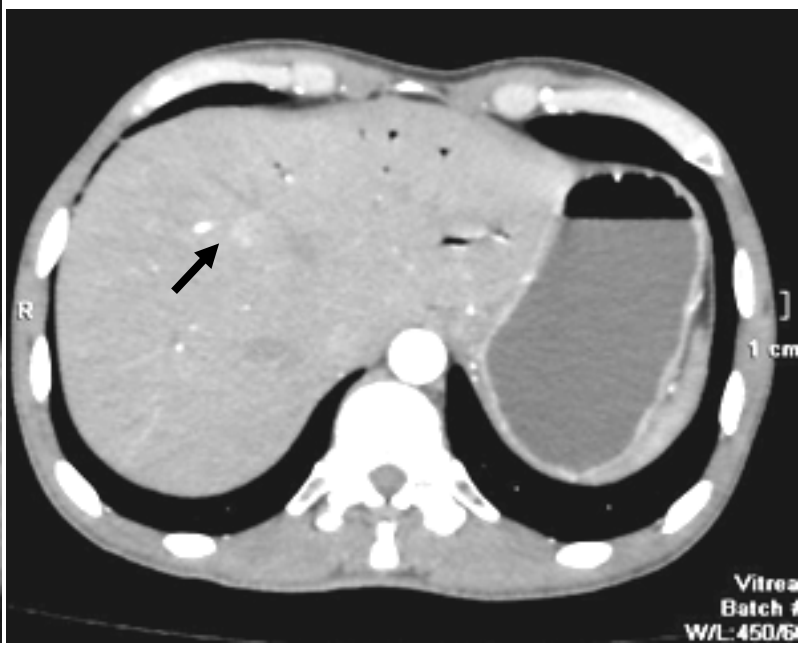

(d) Axial image (arterial phase)

Figure 4. A 34 years old male patient with Jejunal metastasis with lymphadenopathy and hepatic metastasis MDCT showed a focal asymmetric marked wall thickening with submucosal and serosal involvement and heterogenous enhancement forming a $7 \times 5 \times 8.5 \mathrm{~cm}$ mass (a). Associated enlarged mesenteric lymph nodes (white arrow) (b), multiple enlarged porta hepatis lymph nodes, small intestinal obstruction (black arrow) (c) proximal to the mass and a single hepatic focal lesion with early arterial enhancement (black arrow) (d).

The small bowel of this patient showed focal asymmetric marked wall thickening with heterogenous wall enhancement. This thickening involved the submucosa and serosa of the jejunum (Table 6). This secondary jejunal metastasis was associated with enlarged mesenteric lymph nodes, multiple enlarged porta hepatis lymph nodes, single malignant hepatic focal lesion and small intestinal obstruction proximal to the mass.

The pathological biopsy confirmed the MDCT diagnosis and revealed small bowel metastasis secondary to 
Table 5. MDCT criteria of primary duodenal adenocarcinoma.

\begin{tabular}{cccccccc}
\hline $\begin{array}{c}\text { MDCT } \\
\text { criteria } \\
\text { Patients }\end{array}$ & $\begin{array}{c}\text { Pattern of } \\
\text { enhancement }\end{array}$ & $\begin{array}{c}\text { Length of } \\
\text { involvement }\end{array}$ & $\begin{array}{c}\text { Degree of } \\
\text { thickening }\end{array}$ & $\begin{array}{c}\text { Symmetric or } \\
\text { asymmetric thickening }\end{array}$ & $\begin{array}{c}\text { Location along the } \\
\text { small bowel }\end{array}$ & $\begin{array}{c}\text { Location within } \\
\text { the wall }\end{array}$ & $\begin{array}{c}\text { Mesentery and } \\
\text { lymph nodes }\end{array}$ \\
\hline F40Y & heterog & focal & moderate & asymm & duod & Mucosa, Submu & - \\
F50Y & heterog & focal & moderate & asymm & doud & Mucosa-submu. & ------- \\
M55Y & Hetero. & Focal & Moderate & Asymm & Doud & Mucosa-submuc & $\ldots . . . . . . . .$. \\
\hline
\end{tabular}

Table 6. MDCT criteria of secondary jejunal metastasis.

\begin{tabular}{cccccccc}
$\begin{array}{c}\text { MDCT } \\
\text { criteria } \\
\text { Patients }\end{array}$ & $\begin{array}{c}\text { Pattern of } \\
\text { enhancement }\end{array}$ & $\begin{array}{c}\text { Length of } \\
\text { involvement }\end{array}$ & $\begin{array}{c}\text { Degree of } \\
\text { thickening }\end{array}$ & $\begin{array}{c}\text { Symmetric or } \\
\text { asymmetric thickening }\end{array}$ & $\begin{array}{c}\text { Location along the } \\
\text { small bowel }\end{array}$ & $\begin{array}{c}\text { Location within } \\
\text { the wall }\end{array}$ & $\begin{array}{c}\text { Mesentery and } \\
\text { lymph nodes }\end{array}$ \\
\hline M34Y & heterog & focal & marked & asymm & jejunum & Submu, serosa & Mes. L.N. \\
\hline
\end{tabular}

pancreatic adenocarcinoma.

\section{Discussion}

Tumors of the small intestine present a unique challenge to the clinicians across medical specialties. Despite the fact that the small intestine represents approximately $75 \%$ of the total length of the gastrointestinal tract (GI) and more than $90 \%$ of the mucosal surface, and despite its anatomical location between two regions of high cancer risk, the small bowel rarely develops malignant tumors [10]. pproximately 40 different histologic types of both benign and malignant small intestinal tumors have been identified [11].

In Pilleul et al. [9] study, the age range of patients with suspected small-bowel neoplasm was 17 - 98 years with a mean of 53.1 years. While in our study, the age range was $15-70$ years with a mean of 45.5 years. According to Paulsen et al. [12] and Sailer et al. [13], most small bowel tumours are not diagnosed until complications, such as bleeding, bowel obstruction, or perforation, occur. Other clinical symptoms are lassitude, weight loss, and abdominal pain and discomfort. This is confirmed in our study. Our patients presented with abdominal pain, loss of weight, abdominal distention, melena and obstruction. Paulsen et al. [12] also mentioned that the most common small bowel tumors (in decreasing order of frequency of occurrence) were adenocarcinoma, carcinoid tumor, lymphoma, and gastrointestinal stromal tumor. In our study, the most common small bowel tumors were lymphoma, malignant gastrointestinal stromal tumor followed by adenocarcinoma and small bowel metastasis. However, our results were supported by Ghai et al. [14] who stated that lymphoma is the most common malignancy of the small bowel. With the advent of MDCT and the development of 3D image processing, the current role of CT has expanded to the diagnosis and staging of these tumors. When it is difficult to determine their site of origin, 3D imaging may be helpful to better define the site of origin and to help the surgeon plan for resection [4]. Pilleul et al. [9] analyzed the images for the presence of focal bowel wall thickening, small-bowel masses, small-bowel stenosis, mesenteric stranding, enlarged mesenteric lymph nodes, and visceral metastasis. However, we preferred the protocol used by Macari et al. [1] that is the seven previously mentioned criteria. Lymphoma affects the small intestine either as a primary neoplasm that arises focally from the mucosa-associated lymphoid tissue (MALT) or as a part of systemic lymphatic disease [14]. And Lymphoma is almost exclusively of non-Hodgkin type [15]. According to Ghai et al. [14], there is a slight male predilection, Fifteen of our lymphoma patients were males. In our study, four of our patients were under the age of 20 and eight were above the age of 50. This is supported by Ghai et al. [14] who stated that lymphoma occurs predominantly in middle-aged persons ( $6^{\text {th }}$ decade of life) of both sexes, but a double peak can be demonstrated: the first in patients under 10 years of age and the second in patients with a mean age of 53 years. Sailer et al. [13] also added that clinical symptoms are mostly non-specific, and affected patients complain about weight loss, fever, diarrhoea, and non-specific abdominal pain. Our patients complained of recurrent dull-aching abdominal pain, loss of weight, abdominal distention and fever. The MDCT features of lymphoma which was the most common encountered tumor in our study were marked asymmetric segmental wall thickening with homogenous wall enhancement most commonly involving the mucosa and submucosa of the ileum, most commonly associated with enlarged mesenteric lymph nodes. All of these criteria were mentioned in the pattern approach by 
Macari et al. [1] and mentioned in Sailer et al. [13] study as diagnostic MDCT criteria of Lymphoma. Paulsen et al. [12] stated that an exoenteric mass combined with adjacent lymphadenopathy or aneurysmal dilatation suggests lymphoma as the primary consideration. Anzidei et al. [15] also stated that satellite lymphadenopathies are usually bulky, larger than in other neoplasms and may be used as a differential sign. And according to Lee et al. [16], lymphoma is more likely to involve multiple and longer segments of gut and is less likely to cause bowel obstruction. This was confirmed in our study as all lymphoma patients' MDCT showed a mass formation with aneurysmal dilatation without obstruction and most of them were associated with adjacent lymphadenopathy. Gastrointestinal stromal tumor is a common mesenchymal tumour of the gastrointestinal tract, primarily located in the stomach. [13] Malignant gastrointestinal stromal tumor MDCT features in our study were marked asymmetric segmental wall thickening with heterogenous wall enhancement (with necrosis). A pedunculated or predominantly exoenteric mass suggests a gastrointestinal stromal tumor as stated by Paulsen et al. [12]. This is confirmed in our study as all the patients' MDCT showed the formation of exophytic masses. In Sailer et al. [14] study, GIST typically causes liver metastases and peritoneal seeding. Macari et al. [1] stated that adenocarcinoma of the small bowel was located in the proximal small intestine and showed focal moderate asymmetric wall thickening with heterogenous enhancement and with mucosal involvement. Sailer et al. [14] also stated that the duodenum is the most frequently involved site, with the proximal jejunum second and it typically appears as a focal wall thickening involving a short segment that causes luminal stenosis with heterogeneous attenuation and moderate contrast enhancement. This was confirmed in our study as our duodenal adenocarcinoma patient's MDCT showed the same criteria. This was also mentioned in Paulsen et al. [12] study. The jejunal metastasis patient in our study showed focal asymmetric marked wall thickening with heterogenous wall enhancement. This thickening involved the submucosa and serosa of the jejunum and was associated with enlarged mesenteric lymph nodes. In Macari et al. [1] study, these were the criteria of metastatic tumors. Also Sailer et al. [13] stated that advanced stages of pancreatic, biliary, or colonic malignancies tend to infiltrate adjacent small bowel loops and MDCT criteria of metastatic tumors coincided with us.

\section{Conclusion}

Our study has proved that MDCT can be used as a front-line imaging modality for detection of small bowel neoplasms, regarding its ability to show intraluminal, mural and extraintestinal lesions with their characteristic density and features to successfully differentiate between the different neoplasms and sometimes stage them. However, biopsy is still the confirmatory diagnostic method.

\section{Conflict of Interest}

The authors declared no conflict of interest and no financial issues to disclose.

\section{Acknowledgements}

We thank all the Surgical Oncology Department of South Egypt Cancer Institute for their kind cooperation. Also we much appreciate the effort of Radiology Department of South Egypt Cancer Institute and Faculty of Medicine, Assiut University.

\section{References}

[1] Macari, M., Megibow, A. and Balthazar, E. (2007) A Pattern Approach to the Abnormal Small Bowel: Observations at MDCT and CT Enterography. American Journal of Roentgenology, 188, 1344-1355. http://dx.doi.org/10.2214/AJR.06.0712

[2] Patak, M.A., Mortele, K.J., Ros, P.R., et al. (2005) Multidetector Row CT of the Small Bowel. Radiologic Clinics of North America, 43, 1063-1077. http://dx.doi.org/10.1016/j.rcl.2005.07.009

[3] Boudiaf, M., Jaff, A., Soyer, P., et al. (2004) Small-Bowel Diseases: Prospective Evaluation of Multi-Detector Row Helical CT Enteroclysis in 107 Consecutive Patients. Radiology, 233, 338-344. http://dx.doi.org/10.1148/radiol.2332030308

[4] Hong, S.S, Kim, A.Y, Byun, J.W, et al. (2006) MDCT of Small-Bowel Disease: Value of 3 D Imaging. American Journal of Roentgenology, 187, 1212-1221. http://dx.doi.org/10.2214/AJR.04.1762

[5] Maglinte, D.D., Sandrasegaran, K., Lappas, J.C., et al. (2007) CT Enteroclysis. Radiology, 245, 661-671. 
http://dx.doi.org/10.1148/radiol.2453060798

[6] Yu, C.W., Lee, W.J., Tsai, Y.H., et al. (2003) Demonstration of Extensive Mesenteric Venous Thrombosis and Intestinal Infarction with Multidetector Row CT: Value of Curved Planar Reformations. Abdom Imaging, 28, 775-777. http://dx.doi.org/10.1007/s00261-003-0053-7

[7] Jaeckle, T., Stuber, G., Hoffmann, M.H., et al. (2007) Acute Gastrointestinal Bleeding: Value of MDCT. Abdom Imaging, 33, 285-293. http://dx.doi.org/10.1007/s00261-007-9263-8

[8] Liu, Y.B., Liang, C.H., Zhang, Z.L., et al. (2006) Crohn Disease of Small Bowel: Multidetector Row CT with CT Enteroclysis, Dynamic Contrast Enhancement, CT Angiography, and 3D Imaging. Abdom Imaging, 31, 668-674. http://dx.doi.org/10.1007/s00261-006-9092-1

[9] Pilleul F., Penigaud, M., Milot, L., et al. (2006) Possible Small-Bowel Neoplasms: Contrast-Enhanced and WaterEnhanced Multidetector CT Enteroclysis. Radiology, 241, 796-801. http://dx.doi.org/10.1148/radiol.2413051429

[10] Floch, M.H. (2010) Malignant Tumors of the Small Intestine Chapter Published 2010 in Netter's Gastroenterology on Pages 311 to 312. http://dx.doi.org/10.1016/B978-1-4377-0121-0.50126-4

[11] Attanoos, R. and Williams, G.T. (1991) Epithelial and Neuroendocrine Tumors of the Duodenum. Seminars in Diagnostic Pathology, 8, 149-162.

[12] Paulsen, S.R., Huprich, J.E., Fletcher, J.G., et al. (2006) CT Enterography as a Diagnostic Tool in Evaluating Small Bowel Disorders: Review of Clinical Experience with over 700 Cases. Radiographics, 26, 641-657. http://dx.doi.org/10.1148/rg.263055162

[13] Sailer, J., Zacherl, J. and Schima, W. (2007) MDCT of Small Bowel Tumours. Cancer Imaging, 7, 224-233. http://dx.doi.org/10.1102/1470-7330.2007.0032

[14] Ghai, S., Pattison, J., Ghai, S., et al. (2007) Primary Gastrointestinal Lymphoma: Spectrum of Imaging Findings with Pathologic Correlation. Radio Graphics, 27, 1371-1388. http://dx.doi.org/10.1148/rg.275065151

[15] Anzidei, M., Napoli, A., Zini, C., et al. (2011) Malignant Tumours of the Small Intestine: A Review of Histopathology, Multidetector CT and MRI Aspects. The British Journal of Radiology, 84, 677-690. http://dx.doi.org/10.1259/bjr/20673379

[16] Lee, H.J., Im, J.G., Goo, J.M., et al. (2003) Peripheral T-Cell Lymphoma: Spectrum of Imaging Findings with Clinical and Pathologic Features. Radio Graphics, 23, 7-26. http://dx.doi.org/10.1148/rg.231025018 